\title{
PREVENCIÓN DE LA RECURRENCIA PERITONEAL EN EL TRATAMIENTO QUIRÚRGICO DEL CÁNCER GÁSTRICO*
}

\author{
Drs. Marco Ceroni V. ${ }^{1}$, Eduardo Viñuela F. ${ }^{1}$, Enrique Norero M. ${ }^{1}$, Nicolás Jarufe C. ${ }^{1}$, \\ Fernando Crovari E. ${ }^{1}$, Alex Escalona P. ${ }^{1}$, Allan Sharp P. ${ }^{1}$, Eduardo Briceño V. ${ }^{1}$, \\ Cristian Martínez B., Alfonso Díaz F. ${ }^{1}$, Luis Ibáñez A. ${ }^{1}$ \\ 1 Servicio de Cirugía Hospital Sótero del Río. Pontificia Universidad Católica de Chile. \\ Santiago, Chile.
}

\begin{abstract}
\section{Prevention of peritoneal recurrence of gastric cancer}

The peritoneal cavity is the main site of gastric cancer recurrence after curative surgery. When this recurrence occurs, patients may experience bowel obstruction, dehydration and multiple hospital admissions. The therapeutic options that may decrease the rate of peritoneal recurrence and increase five years survival are intraoperative hyperthermic chemotherapy, extensive intraoperative peritoneal lavage and routine bursectomy. We herein review the oncological results of curative surgery for gastric cancer, its failure patterns and the risk factors for peritoneal recurrence. We also review the studies aiming to prevent peritoneal carcinomatosis.

Key words: Gastric cancer, peritoneal recurrence, carcinomatosis.
\end{abstract}

\section{Resumen}

La recurrencia peritoneal en el cáncer gástrico avanzado, luego de una cirugía con intención curativa es un problema que debe ser considerado una prioridad en nuestro país, debido a la alta proporción de pacientes con compromiso de la serosa gástrica al momento del diagnóstico, siendo el peritoneo el principal sitio de recurrencia luego de una cirugía con intención curativa en este grupo. Luego de la recurrencia peritoneal, los pacientes presentan un deterioro importante de la calidad de vida, principalmente por episodios de obstrucción intestinal, deshidratación y rehospitalizaciones. Se han realizado múltiples estudios en el extranjero de potenciales medidas que se pueden realizar durante la cirugía inicial con intención curativa que previenen la recurrencia. Algunas de estas medidas han disminuido la recurrencia peritoneal y aumentado la sobrevida a 5 años, como son la quimioterapia hipertérmica intra-operatoria, el lavado peritoneal extensivo y la bursectomía rutinaria, en pacientes seleccionados. El objetivo de la presente revisión es mostrar los resultados oncológicos de la cirugía curativa, los patrones de falla luego de una cirugía curativa, los factores de riesgo para una recurrencia peritoneal y los estudios que se han realizado en relación a la prevención de la carcinomatosis peritoneal.

Palabras clave: Cáncer gástrico, cirugía curativa, recurrencia peritoneal, prevención.

\footnotetext{
Los autores no refieren conflictos de interés.

Correspondencia: Dr. Marco Ceroni V. marco.ceroni@gmail.com
}

*Recibido el 21 de octubre de 2013 y aceptado para publicación el 23 de noviembre de 2013. 


\section{Introducción}

El cáncer gástrico $(\mathrm{CG})$ es la primera causa de muerte oncológica en Chile ${ }^{1}$. La mayoría de los pacientes en nuestro país son diagnosticados en etapas avanzadas de esta enfermedad (85 a 90\%) y un pequeño porcentaje son tumores incipientes. Los esfuerzos actuales en el CG deben estar enfocados en 2 aspectos para mejorar los resultados oncológicos y la sobrevida: 1) Aumentar el hallazgo endoscópico de tumores incipientes, ya que es posible en esta etapa, la curación de esta enfermedad; 2) Disminuir la recurrencia peritoneal posterior a la cirugía con intención curativa en el CG avanzado.

La gastrectomía curativa (R0) en Chile, aún presenta resultados de sobrevida bajos, los cuales se encuentran entre el 20 y el $60 \%$ a 5 años de seguimiento, según el estadio tumoral ${ }^{2,3}$, al observar las curvas de sobrevida a 5 años en pacientes con compromiso de la serosa gástrica, los pacientes tienen una sobrevida a 5 años de $34 \%{ }^{4}$.

Los resultados de sobrevida a 5 años en la literatura para las series de pacientes tratados con gastrectomía con disección linfática de la segunda barrera ganglionar (D2) R0, demuestran que en occidente la sobrevida global se encuentra alrededor del $40 \% \%^{5-9}$ y en países orientales sobre el $60 \%{ }^{10-13}$, esto posiblemente por 3 motivos: el primero, es que los grupos quirúrgicos en el oriente excluyen del reporte a aquellos pacientes con citología positiva intra-operatoria, debido a que se asocia a un peor pronóstico; lo segundo, es que el estadio final del análisis patológico de la pieza operatoria tiene una proporción mayor de pacientes con cánceres incipientes en los grupos orientales y lo tercero, es que aparentemente hay una diferencia genética entre ambos grupos ${ }^{14}$ (Tablas 1 y 2 ).

Para calcular con mayor precisión el pronóstico de un paciente con CG operado con intención curativa con disección linfática D2, es útil el uso de un nomograma validado, el que fue establecido por Han en base a 7.954 pacientes $^{15}$. Según este nomograma, un paciente tratado en nuestro país, frecuentemente tendrá a 5 años una sobrevida de $25 \%$ y a 10 años de $15 \%$.

Actualmente, en Chile no hay duda, que la cirugía ideal es la gastrectomía total con disección linfática D2, similar a lo que se realiza en Japón. Los resultados quirúrgicos nacionales son buenos en relación a la morbi-mortalidad postoperatoria ${ }^{16}$, sin embargo, el gran problema actual, es el alto porcentaje de recurrencia después de una cirugía con intención curativa, la que ocurre dentro de los 2 a 4 años de seguimiento ${ }^{17}$. Una vez que ocurre la recurrencia, la sobrevida a 2 años es inexistente.

Los lugares de recurrencia en pacientes tratados con gastrectomía con intención curativa varían principalmente según la extensión de la disección

Tabla 1. Sobrevida a 5 años de los grupos de pacientes con cirugía D2 en países occidentales, se detalla los porcentajes de la profundidad del tumor de la biopsia definitiva (*)

\begin{tabular}{|crlcccc|}
\hline $\begin{array}{c}\text { Sobrevida } \\
\text { a 5 años }\end{array}$ & n & Autor-año & $\begin{array}{c}\text { Mucosa o } \\
\text { submucosa* }\end{array}$ & $\begin{array}{c}\text { Muscular o } \\
\text { subserosa* }\end{array}$ & Serosa* & $\begin{array}{c}\text { Órganos } \\
\text { vecinos* }\end{array}$ \\
\hline $33 \%$ & 195 & UK trial 1998 & $21 \%$ & $35 \%$ & $44 \%$ & $0 \%$ \\
$48 \%$ & 328 & Dutch trial 1999 & $26 \%$ & $46 \%$ & $25 \%$ & $3 \%$ \\
$40,1 \%$ & 1.038 & Karpeh M. 2000 & $18 \%$ & $30 \%$ & $49 \%$ & $3 \%$ \\
$59 \%$ & 82 & Edward P. 2004 & $9 \%$ & $16 \%$ & $59 \%$ & $17 \%$ \\
$50 \%$ & 65 & EORT-TC trial 40954 $2010^{7}$ & $6 \%$ & $46 \%$ & $37 \%$ & $10 \%$ \\
\hline
\end{tabular}

Tabla 2. Sobrevida a 5 años de los grupos de pacientes con cirugía D2 en países orientales, se detalla los porcentajes de la profundidad del tumor de la biopsia definitiva (*)

\begin{tabular}{|cclcccc|}
\hline $\begin{array}{c}\text { Sobrevida } \\
\text { a 5 años }\end{array}$ & n & Autor-año & $\begin{array}{c}\text { Mucosa o } \\
\text { submucosa* }\end{array}$ & $\begin{array}{c}\text { Muscular o } \\
\text { subserosa* }\end{array}$ & $\begin{array}{c}\text { Serosa* } \\
\text { Órganos } \\
\text { vecinos* }\end{array}$ \\
$51,3 \%$ & 121 & Wu CW. 2006 & $24 \%$ & $16 \%$ & $49 \%$ & $2 \%$ \\
$69,2 \%$ & 263 & Sasako M. 2008 (JCOG trial 9501$)^{11}$ & $3 \%$ & $47 \%$ & $46 \%$ & $3 \%$ \\
$61,1 \%$ & 530 & Sasako M. (ACTS-GC) 2011 & $54 \%$ & $43 \%$ & $3 \%$ & $0 \%$ \\
$60,9 \%$ & 133 & JCOG9206-2 2011 & $2 \%$ & $29 \%$ & $66 \%$ & $3 \%$ \\
\hline
\end{tabular}


Tabla 3. Patrones de recurrencia, se observa que las series del oriente (3 primeras filas) tienen mayor porcentaje de recurrencia peritoneal y las series del occidente mayor porcentaje de recurrencia local. Con el texto en negrita están las series con mayor cantidad de pacientes

\begin{tabular}{|c|c|c|c|c|c|}
\hline & $\mathbf{n}$ & Local & Hepática & Distancia & Peritoneal \\
\hline Yoo CH. $2000^{18}$ & $652 / 2.328$ & $33 \%$ & $19 \%$ & $21 \%$ & $44 \%$ \\
\hline Maehara Y. $2003^{50}$ & $207 / 939$ & $22 \%$ & $33 \%$ & $21 \%$ & $43 \%$ \\
\hline Sasako M. (JCOG trial 9501) $2008^{11}$ & $215 / 523$ & $22 \%$ & $21 \%$ & $19 \%$ & $38 \%$ \\
\hline D'Angelica M. $2004^{17}$ & $496 / 1.172$ & $40 \%$ & $18 \%$ & $20 \%$ & $22 \%$ \\
\hline Roviello F. $2003^{21}$ & $215 / 441$ & $45 \%$ & $27 \%$ & $9 \%$ & $36 \%$ \\
\hline
\end{tabular}

linfática, los reportes muestran que los pacientes con una disección linfática D2 presentarán con mayor frecuencia una recurrencia peritoneal, como ocurre en países orientales ${ }^{18}$, con mayor recurrencia local en pacientes a los que no se les realizó una disección $\mathrm{D} 2{ }^{17}$. Al realizar una cirugía con disección linfática, como la que se realiza en Japón, con mayor probabilidad tendremos una recurrencia peritoneal en nuestros pacientes (Tabla 3 ).

Se debe tener en consideración que el diagnóstico del patrón de recurrencia de los trabajos citados no es tan preciso, debido a que a un grupo importante de pacientes se realizó el diagnóstico de la recurrencia en base a estudios radiológicos, sin confirmación patológica, lo que está asociado a un porcentaje de error por su baja sensibilidad. En contraste con esto, a finales de los años 70, algunos grupos plantearon realizar una re-exploración programada para pacientes con CG tratados con intención curativa, para determinar si era posible tratar en forma quirúrgica una recurrencia precoz, sin embargo, esta idea en CG fue abandonada, debido a los malos resultados oncológicos. La información relevante que aportaron estos trabajos es que determinaron el patrón preciso de recurrencia peritoneal, el cual es de $50 \%{ }^{19}$.

El objetivo de la presente revisión es mostrar la evidencia actual en relación a medidas para la prevención de la recurrencia peritoneal en pacientes con $\mathrm{CG}$ avanzado tratados con gastrectomía con intención curativa.

Para entender las medidas que disminuyen la recurrencia peritoneal, es necesario conocer los factores de riesgo, marcadores genéticos, los mecanismos de diseminación, la fisiopatología de los implantes peritoneales y el concepto de barrera peritoneocirculación sistémica.

\section{Factores de riesgo para carcinomatosis peritoneal}

Los factores de riesgo asociados a recurrencia peritoneal, después de una cirugía con intención curativa son el tipo histopatológico difuso de Lauren $^{17,20,21}$, el compromiso de la serosa gástrica ${ }^{17}$, una disección linfática D2 ${ }^{18}$, la ubicación del tumor en el antro gástrico ${ }^{17}$, el compromiso ganglionar ${ }^{18}$, los tumores indiferenciados ${ }^{17}$, el sangrado intraoperatorio y la necesidad de transfusiones en el periodo postoperatorio $^{22}$.

\section{Marcadores genéticos de recurrencia peritoneal}

Se han identificado varios marcadores genéticos del lavado peritoneal que son específicos para células tumorales, a diferencia del mRNA de antígeno carcinoembrionario (CEA), los cuales tienen la capacidad de predecir el riesgo de recurrencia peritoneal, incluso en pacientes con citología convencional negativa. Al ser positivos con PCR transcriptasa reversa para CK20, FABP1 y MUC2, los pacientes tienen un riesgo, respectivamente, de 50\%, 67\% y $71 \%$ de recurrencia peritoneal a los 2,5 años. Si se encuentran los tres marcadores negativos, el riesgo de recurrencia es menor al $5 \%{ }^{23}$. La oncoproteína fetal IMP-3 predice en forma similar al CEA el riesgo de recurrencia peritoneal ${ }^{24}$, por lo que no sería tan útil.

\section{Patrones de diseminación}

Los cuatro patrones clásicos de diseminación del CG son el crecimiento local, el celómico, el hematógeno y el linfático. Debido a la cirugía se produce un $5^{\circ}$ mecanismo secundario al trauma quirúrgico en que se liberan células neoplásicas en forma directa a la cavidad abdominal de la pieza operatoria, de los conductos linfáticos y de los vasos sanguíneos (Figura 1). Esto explica en parte la razón por lo que la recurrencia peritoneal es frecuente. Un trabajo realizado por Marutsuka ${ }^{25}$, midió la cantidad de células neoplásicas previo y posterior a la disección linfática y demostró que incluso los pacientes con $\mathrm{CG}$ con compromiso de la submucosa tienen una 
citología positiva posterior a la etapa resectiva, alcanzando un $14,3 \%$.

\section{Teoría de los implantes peritoneales}

Según la teoría del Dr. Surgarbaker ${ }^{26}$, el desarrollo de un implante peritoneal ocurre en dos etapas: 1) Liberación de células neoplásicas y 2) Atrapamiento de dichas células por el proceso inflamatorio. La liberación de las células neoplásicas puede ocurrir en dos situaciones según el compromiso de la serosa gástrica: 1) En pacientes sin compromiso de la serosa gástrica, las células neoplásicas son liberadas a la cavidad abdominal por manipulación del tumor y/o liberación de células neoplásicas de canales linfáticos y de los vasos sanguíneos cortados durante el procedimiento y 2) en pacientes con compromiso seroso, además de lo anterior, exudación de células neoplásicas previo a la cirugía. La segunda etapa es el atrapamiento de las células neoplásicas, un ejemplo característico es el desarrollo del tumor de Krunkenberg: el cuerpo hemorrágico del ovario produce un proceso inflamatorio, que permite la implantación de células tumorales. En la segunda etapa

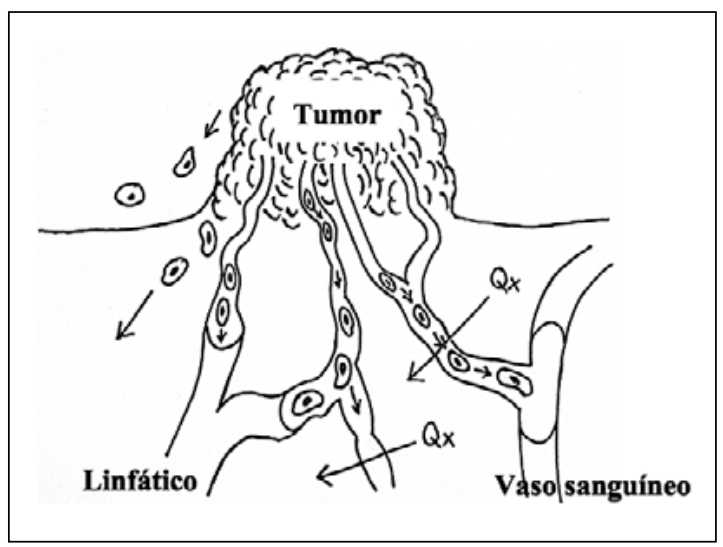

Figura 1. Patrones de diseminación. ocurre algo parecido, las células neoplásicas quedan atrapadas en la fibrina, se produce un aumento de células inflamatorias y de factores de crecimiento, que favorecen la formación de un implante peritoneal (Figura 2).

\section{Barrera peritoneo-circulación sistémica}

Este concepto es importante, el peritoneo es una monocapa de células mesoteliales, los vasos sanguíneos se encuentran alejados de esta capa ( 90 um), esta sería la razón por la cual, la quimioterapia sistémica no ha mostrado regresión completa de los implantes peritoneales, ni previene la recurrencia peritoneal $^{27,28}$, ya que se tendrían que utilizar altas dosis de quimioterapia ${ }^{29}$, en niveles tóxicos, para llegar al peritoneo y tener algún grado de respuesta. Por lo que es más lógico pensar en tratamientos adyuvantes a través de la cavidad peritoneal, en que el paso de la quimioterapia a la circulación sistémica sea mínimo.

\section{Prevención de la carcinomatosis peritoneal en cáncer gástrico}

Las medidas descritas en la literatura para la prevención de la carcinomatosis peritoneal en el cáncer gástrico se dividen en preoperatorias, intraoperatorias y postoperatorias. Previo a la cirugía está el mejorar el estado nutricional del paciente, esto está relacionado en forma indirecta a la prevención de la recurrencia peritoneal, en que se mejora el estado inmunológico y como consecuencia se disminuyen las complicaciones; La segunda medida descrita es la quimioterapia intraperitoneal neoadyuvante. Durante la cirugía, la mayoría de las medidas utilizadas corresponden a principios oncológicos clásicos: la resección de la pieza operatoria en bloque, la manipulación mínima del tumor, la hemostasia prolija, el control de los tiempos quirúrgicos y algunas que han

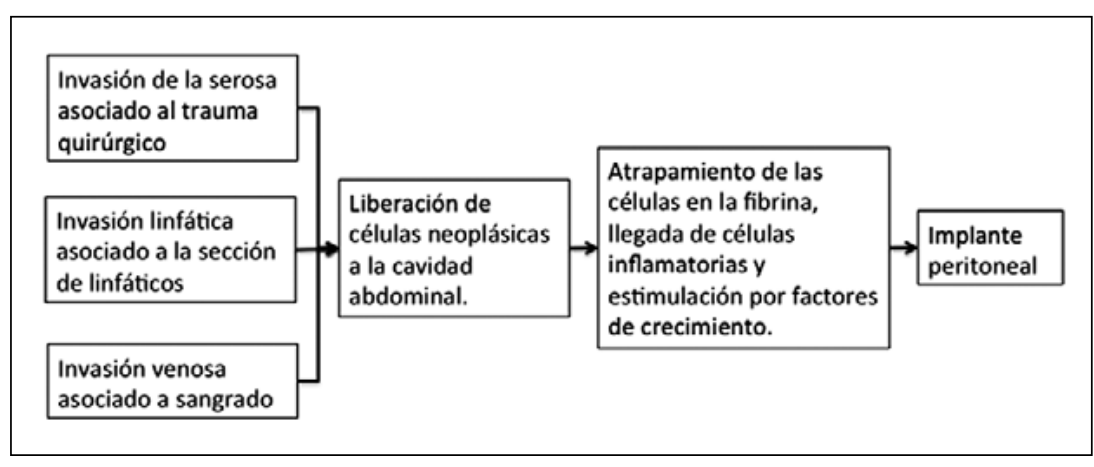

Figura 2. Desarrollo de un implante peritoneal. 
surgido en el último tiempo, como es la bursectomía rutinaria, la quimioterapia intraperitoneal, el lavado peritoneal extensivo y la gastrectomía laparoscópica. Posterior a la cirugía se debe evitar las transfusiones de sangre y la quimioterapia intraperitoneal adyuvante. También existen estudios experimentales con virus en ratones que impiden la implantación de las células neoplásicas en la cavidad abdominal, sin embargo, aun no existen estudios en humanos ${ }^{30}$. A continuación se describen las medidas principales estudiadas para la prevención de la recurrencia peritoneal.

\section{1) Quimioterapia intraperitoneal neoadyuvante}

En la literatura, se ha realizado solo un estudio, el que incluyó 24 pacientes con CG con compromiso seroso, confirmado por laparoscopía, excluidos los pacientes con carcinomatosis peritoneal, con metástasis a distancia o con CG incipiente. Durante la laparoscopía se dejó un drenaje subdiafragmático izquierdo y otro en el fondo de saco rectovesical. El esquema de quimioterapia intraperitoneal neoadyuvante consistió en Mitomicina $\mathrm{C}$ el primer día, cinco días de Cisplatino. Se realizó la gastrectomía 2 a 4 semanas después. Se observó disminución de la profundidad del tumor, sólo un paciente con toxicidad tipo 3 por la quimioterapia y un $45 \%$ de recurrencia peritoneal ${ }^{31}$. Sin embargo, no se puede determinar si hay una disminución de la recurrencia peritoneal, ya que no tiene un grupo control.

\section{2) Bursectomía}

La bursectomía es considerada parte de la cirugía estándar en Japón desde 1960, la cual se realiza cada vez menos, debido al aumento de pacientes en etapas más tempranas de esta enfermedad. Consiste en la resección del peritoneo que se encuentra sobre la cara superior del mesocolon transverso y superficie anterior al páncreas (Figura 3). Su efecto oncológico se ha confirmado en pacientes con cáncer gástrico con compromiso de la serosa hacia la cara posterior del estómago ${ }^{32}$. Un estudio randomizado prospectivo reciente $^{33}$, demostró que la bursectomía prolonga la cirugía en 26 minutos, tiene mayor riesgo de sangrado (475 cc vs $350 \mathrm{cc} \mathrm{p}=0,047)$, sin aumento de la morbilidad $(14,3 \%)$, ni mortalidad $(0,95 \%)$ postoperatoria. Disminuye la recurrencia peritoneal de $13,2 \%$ a $8,6 \%$ y en el grupo de pacientes con compromiso de la serosa gástrica, tiene una tendencia a mejorar la sobrevida global y libre de enfermedad a 5 años, sin significación estadística $(\mathrm{p}=0,08)$.

\section{3) Quimioterapia intraperitoneal}

Este tratamiento adyuvante se indicó por $1^{\circ}$ vez en 1950 por Weisberger para la ascitis de origen maligno $^{34}$, actualmente tiene efecto terapéutico demostrado para el cáncer de ovario en 8 estudios randomizados controlados ${ }^{35}$, y para tratamiento complementario posterior a la citoreducción en el cáncer de colon en un estudio randomizado contro$\operatorname{lado}^{36}$. La teoría de su efectividad se debe a que se logran dosis más elevadas de quimioterapia en el peritoneo, en comparación con el uso intravenoso, disminuyendo los efectos tóxicos sistémicos. Tiene múltiples controversias en relación a su uso: Real indicación, quimioterapia de elección, dosis, el uso de hipertermia, volumen de infusión, tiempo de tratamiento, efectividad y complicaciones.

Existen varias siglas utilizadas en la literatura determinadas por el momento de indicación en relación a la cirugía y el uso de hipertermia. La HIIC o HIPEC corresponde a la quimioterapia intraperitoneal intraoperatoria hipertérmica, la NIIC es la quimioterapia intraperitoneal normotérmica, la EPIC es la quimioterapia intraperitoneal en el periodo postoperatorio temprano y la DPIC es el esquema de quimioterapia intraperitoneal postoperatoria diferido.

En cuanto a la farmacología, el principio es maximizar la dosis de quimioterapia en la cavidad peritoneal, disminuyendo el paso a la circulación sistémica y así disminuir los efectos tóxicos sistémicos. El

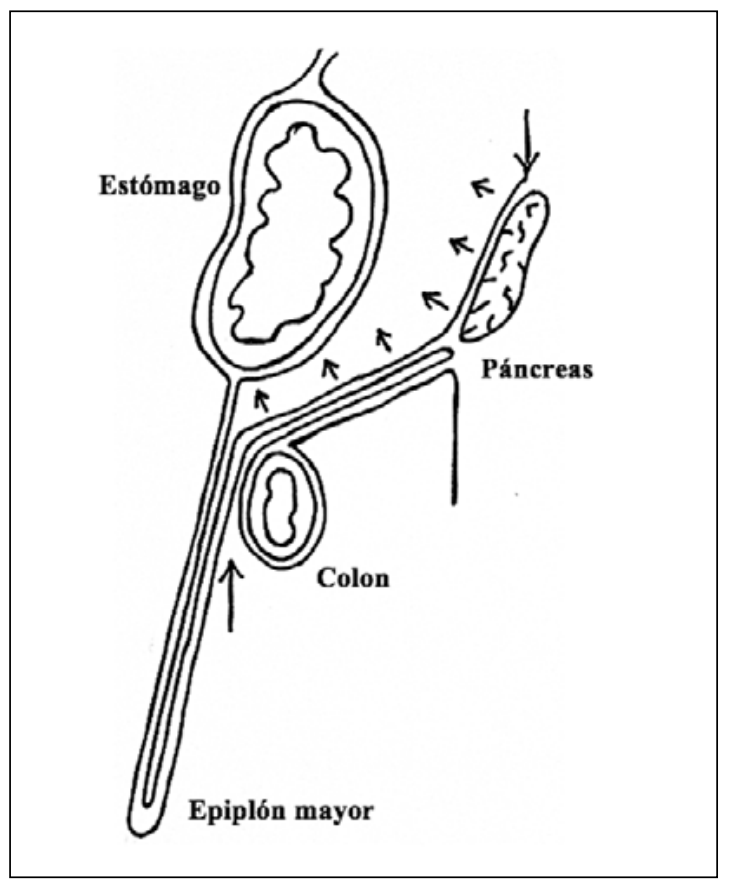

Figura 3. Técnica de bursectomía. Las flechas pequeñas indican la resección de la hoja posterior de la transcavidad de los epiplones para realizar la bursectomía. Las flechas grandes indican el plano avascular, que se debe seguir para la busersectomía. 
efecto de la quimioterapia depende de la capacidad de la droga de difundir en el tumor, la concentración en la solución y la tasa de paso a la circulación sistémica. Las características ideales para mejorar su efectividad es que sea hidrofílica, ionizante y de mayor peso molecular posible. Se han estudiado que las diferentes drogas utilizadas son capaces de difundir entre 2 a $3 \mathrm{~mm}$, aumentando el efecto de algunas drogas con la hipertermia $\left(\mathrm{T}=43^{\circ} \mathrm{C}\right)^{37}$. Los fármacos más indicados, son la mitomicina $\mathrm{C}$ sola $\mathrm{o}$ asociada a cisplatino. El cisplatino tiene la característica de aumentar su efecto con la hipertermia, la doxorubicina tiene una excelente tasa de relación de concentración peritoneo/circulación sistémica y además, un efecto esclerosante, por lo que es útil en pacientes con ascitis maligna invalidante ${ }^{38}$. El paclitaxel se utiliza con mayor frecuencia en el periodo postoperatorio, es el fármaco con mayor peso molecular, por lo que se debe movilizar al paciente para su distribución uniforme.

En el año 2007 se realizó una revisión sistemática y metaanálisis ${ }^{39}$, de todos los estudios randomizados controlados en los cuales se indicó quimioterapia intraperitoneal adyuvante posterior a la cirugía, la que se comparó con la cirugía exclusiva. Se incluyeron en el análisis 13 estudios, en los cuales se indicaron distintos esquemas de quimioterapia (HIPEC, NIIH, EPIC, DPIC), algunos asociados a quimioterapia sistémica. La quimioterapia más utilizada fue la mitomicina $\mathrm{C}$ asociada a cisplatino, con un volumen de infusión de 4 litros, a una temperatura entre $41^{\circ}$ $43^{\circ} \mathrm{C}$. La sobrevida global fue mayor en el grupo de quimioterapia intraperitoneal en comparación con la cirugía exclusiva. En el meta-análisis se observó diminución de la recurrencia peritoneal solo con la quimioterapia en el periodo postoperatorio precoz (EPIC), sin embargo, al incluir los dos estudios que se consignó la recurrencia sólo en los pacientes fallecidos, la HIPEC disminuyó la recurrencia peritoneal de 59 a $39 \%{ }^{40}$, y en el otro de 48 a $5 \%{ }^{41}$. Globalmente, la quimioterapia intraperitoneal, independiente del esquema, no presenta aumento de las complicaciones, ni de la mortalidad. No se asocia a aumento de dehiscencia de anastomosis, ni fístulas. Sin embargo, aumenta la cantidad de colecciones en $137 \%$ y neutropenia en $333 \%$. Esto último demuestra que habría un paso de la quimioterapia a la circulación sistémica.

El meta-análisis más reciente comparó la HIPEC asociado a cirugía con la cirugía exclusiva en el tratamiento curativo del CG, se observó en 16 estudios, que incluyeron un total de 1.906 pacientes, que la HIPEC mejora la sobrevida a 5 años, reduce la recurrencia peritoneal, no tiene más riesgo de filtraciones, íleo, perforación intestinal, ni mielosupresión. Sólo aumenta el dolor abdominal postoperatorio ${ }^{42}$.

\section{Técnica de HIPEC}

Es una técnica que consiste, una vez terminada la fase resectiva y antes de la reconstrucción, en instalar 4 tubos en la cavidad abdominal, dos conectados a una bomba que lleva la solución con la quimioterapia a la cavidad abdominal (uno subdiafragmático derecho y otro hacia el borde de la herida operatoria) $\mathrm{y}$ dos drenajes (uno subdiafragmático izquierdo $\mathrm{y}$ uno en el fondo de saco). Todos los tubos se conec$\tan$ a intercambiadores térmicos y se mide la temperatura en forma continua, la cual debe permanecer constante entre $41^{\circ}$ y $43^{\circ} \mathrm{C}$. La infusión re-circulante debe permanecer a un flujo de $1 \mathrm{lt} / \mathrm{min}$.

\section{Resumen de la quimioterapia intraperitoneal adyuvante}

El fármaco más utilizado es la mitomicina $\mathrm{C}$, asociado a cisplatino, el efecto de la hipertermia aumenta la sobrevida. Esta técnica, no tiene más mortalidad, ni fístulas, no está exenta de complicaciones (abscesos y neutropenia) y los esquemas que demostraron disminución de la recurrencia peritoneal son el HIPEC y el EPIC.

\section{4) Lavado peritoneal extensivo (EILP) a $41^{\circ} \mathrm{C}$}

Consiste en el lavado con 10 litros de suero fisiológico a $41^{\circ} \mathrm{C}$ después de la fase resectiva. Fue propuesto por Kuramoto ${ }^{43}$, el cual realizó un estudio prospectivo randomizado con 3 grupos; el primero tuvo cirugía exclusiva, el segundo grupo cirugía asociado a quimioterapia normotérmica intraoperatoria con cisplatino y un tercer grupo cirugía, quimioterapia normotérmica intraoperatoria, asociado a EILP. Se incluyeron solo los pacientes con citología intraoperatoria positiva. Se observó que el grupo con EILP tuvo una sobrevida a 5 años de $43,8 \%$, mayor a los otros dos grupos, con una diferencia de casi $40 \%$. Con una disminución de la recurrencia peritoneal en forma significativa de $80-90 \%$ a $40 \%$. La teoría se basa en la dilución de las células neoplásicas liberadas a la cavidad abdominal durante la fase resectiva a cero. Esto se realiza con suero fisiológico, con un litro cada vez, se movilizan las asas intestinales y se aspira el contenido, repitiendo el procedimiento 10 veces. Los autores postulan que los resultados se deben a la dilución de las células neoplásicas, sin embargo, durante el lavado utilizaron suero fisiológico a $41^{\circ} \mathrm{C}$, por lo que habría más de un mecanismo involucrado (dilución e hipertermia). El mismo grupo realizó un estudio con citología con detección de mRNA de CEA y de CK20 con PCR-ultra rápida, el cual demostró que después de 8 lavados, la cantidad de células neoplásicas disminuyen a $0^{25}$. Estos resultados también fueron confirmados en un grupo de pacientes en China, con gastrectomía total 
D2, con citología al inicio, al término de la resección y posterior al lavado peritoneal extensivo ${ }^{44}$. Con el mismo tratamiento se ha demostrado disminución de la recurrencia peritoneal en el cáncer de páncreas, sin embargo, sin aumento de la sobrevida, debido a la biología más agresiva de este cáncer ${ }^{45}$; también se ha demostrado con esta técnica, el aumento de la sobrevida en el hepatocarcinoma roto ${ }^{46} \mathrm{y}$ en estudios experimentales en cáncer de colon ${ }^{47}$.

\section{5) Gastrectomía laparoscópica}

La gastrectomía laparoscópica tiene claros beneficios en la recuperación postoperatoria de los pacientes, disminuye el trauma quirúrgico de una laparotomía, mantiene un mejor estado inmunológico y tiene un menor desgaste metabólico en comparación con el abordaje abierto ${ }^{48}$. Existe controversia en relación a si aumenta el riesgo de desarrollar implantes en los sitios de los puertos por vía transcelómica. En relación a esto, Ying realizó un estudio prospectivo, que comparó el abordaje laparoscópico con el abierto y observó que el porcentaje de positivización de la citología antes y después de la etapa resectiva, en ambos grupos aumentaba en $6 \%$, sin diferencia estadísticamente significativa ${ }^{49}$. Por lo que el abordaje laparoscópico es tan seguro como el abierto, en el tratamiento quirúrgico del CG.

En resumen, las medidas demostradas que previenen la recurrencia peritoneal en el tratamiento quirúrgico del CG son: 1) La bursectomía rutinaria en pacientes con compromiso de la serosa; 2) la quimioterapia intraperitoneal adyuvante; 3) el lavado peritoneal extensivo. Estas medidas deben ser valoradas y estudiadas para determinar en forma consistente la prevención de la recurrencia peritoneal en nuestro país.

\section{6) Prevención de la recurrencia peritoneal en el cáncer de colon}

Un modelo interesante realizado en cáncer de colon, se puede aplicar en forma similar a los pacientes con CG. Existe un subgrupo de pacientes con cáncer de colon que presentan mayor riesgo de recurrencia peritoneal, que son aquellos pacientes con presencia de mucina o con células en anillo de sello. Sammmartino $0^{50}$, realizó un estudio para la prevención de recurrencia peritoneal en que se incluyeron este tipo de pacientes, sin metástasis y en buen estado funcional. Realizó la colectomía, la omentectomía completa, la anexectomía en pacientes postmenopáusicas, la resección del ligamento redondo, la apendicectomía e HIPEC con oxaliplatino y los compararon con el tratamiento estándar. Observó que este tratamiento no aumenta la morbilidad, la mortalidad, el tiempo quirúrgico, sangrado intraoperatorio, las complicaciones, ni los días de hospitalización. No aumentó la sobrevida global, pero sí en forma significativa la sobrevida libre de enfermedad. Y disminuyó la recurrencia peritoneal de $22 \%$ a $4 \%(p<0,05)$.

En resumen, la recurrencia peritoneal en el tratamiento quirúrgico curativo del CG avanzado es frecuente, se conocen actualmente mediadas intraoperatorias efectivas para su prevención, como es la quimioterapia hipertérmica, el lavado peritoneal extensivo y la bursectomía. Aún faltan estudios que reproduzcan los resultados en un mayor grupo de pacientes, como es la técnica del lavado peritoneal extensivo.

\section{Referencias}

1. Guía GES Cáncer gástrico 2010 .

2. García CC, Benavides CC, Apablaza SP, Rubilar PO, Covacevich SR, Peñaloza PM y cols. Resultados del tratamiento quirúrgico del cáncer gástrico. Análisis de 423 casos. Rev Med Chile 2007;135:687-95.

3. Pimentel F, Guzmán S, Llanos O, Rahmer A, Zúñiga A, Meissner A. Cáncer gástrico: Resultados Quirúrgicos y Sobrevida. Rev Med Chile 1988;116:525-31.

4. Astete G, Lynch O, Madariaga J, Zilic M, Martínez L, Schultz H y cols . Patrón pronóstico de invasión serosa en cáncer gástrico. Rev Chil Cir. 2002;54:644-8.

5. Cuschieri A, Weeden S, Fielding J, Bancewicz J, Craven J, Joypaul V, et al. Patient survival after D1 and D2 resections for gastric cancer: long-term results of the MRC randomized surgical trial. British Journal of Cancer 1999;79:1522-30.

6. Bonenkamp JJ, Hermans J, Sasako M, van de Velde CJ, Welvaart K, Songun I, et al; Dutch Gastric Cancer Group. Extended lymph-node dissection for gastric cancer. N Engl J Med. 1999;340:908-14.

7. Karpeh MS, Leon L, Klimstra D, Brennan MF. Lymph node staging in gastric cancer: is location more important than Number? An analysis of 1.038 patients. Ann Surg. 2000;232:362-71.

8. Edwards P, Blackshaw GR, Lewis WG, Barry JD, Allison MC, Jones DR. Prospective comparison of D1 vs modified D2 gastrectomy for carcinoma. British Journal of Cancer 2004;90:1888-92.

9. Schuhmacher C, Gretschel S, Lordick F, Reichardt P, Hohenberger W, Eisenberger CF, et al. Neoadjuvant chemotherapy compared with surgery alone for locally advanced cancer of the stomach and cardia: European Organization for Research and Treatment of Cancer randomized trial 40954. J Clin Oncol. 2010;28:5210-8.

10. Wu CW, Hsiung CA, Lo SS, Hsieh MC, Chen JH, Li $\mathrm{AF}$, et al. Nodal dissection for patients with gastric cancer: a randomised controlled trial. Lancet Oncol. 2006;7:309-15.

11. Sasako M, Sano T, Yamamoto S, Kurokawa Y, Nashimoto A, Kurita A, et al; Japan Clinical Oncology 
Group. D2 lymphadenectomy alone or with para-aortic nodal dissection for gastric cancer. New Engl J Med. 2008;359:453-62.

12. Sasako M, Sakuramoto S, Katai H, Kinoshita T, Furukawa $\mathrm{H}$, Yamaguchi $\mathrm{T}$, et al. Five-year outcomes of a randomized phase III trial comparing adjuvant chemotherapy with S-1 versus surgery alone in stage II or III gastric cancer. J Clin Oncol. 2011;29:4387-93.

13. Miyashiro I, Furukawa H, Sasako M, Yamamoto S, Nashimoto A, Nakajima S, et al; Gastric Cancer Surgical Study Group in the Japan Clinical Oncology Group. Randomized clinical trial of adjuvant chemotherapy with intraperitoneal and intravenous cisplatin followed by oral fluorouracil (UFT) in serosa-positive gastric cancer versus curative resection alone: final results of the Japan Clinical Oncology Group trial JCOG9206-2. Gastric Cancer. 2011;14:212-8.

14. Bickenbach K, Strong V. Comparisons of gastric cancer treatments: east vs west. J Gastric Cancer 2012;12:5562.

15. Han DS, Suh YS, Kong SH, Lee HJ, Choi Y, Aikou $\mathrm{S}$, et al. Normogram predicting long-term survival after D2 gastrectomy for gastric cancer. J Clin Oncol. 2012;30:3834-40.

16. Butte JM, Kerrigan N, Waugh E, Meneses M, Parada $\mathrm{H}$, Visscher A y cols. Gastrectomía extendida en cáncer gástrico. Evaluación de la morbilidad y mortalidad postoperatoria. Rev Med Chile 2010;138:1487-94.

17. D'Angelica M, Gonen M, Brennan MF, Turnbull AD, Bains M, Karpeh MS. Patterns of initial recurrence in completely resected gastric adenocarcinoma. Ann Surg. 2004;240:808-16.

18. Yoo CH, Noh SH, Shin DW, Choi SH, Min JS. Recurrence following curative resection for gastric carcinoma. Br J Surg. 2000;87:236-42.

19. Gunderson LL, Sosin H. Adenocarcinoma of the stomach: areas of failure in a re-operation series (second or symptomatic look) clinicopathologic correlation and implications for adjuvant therapy. Int J Radiat Oncol Biol Phys. 1982;8:1-11.

20. Marrelli D, Roviello F, de Manzoni G, Morgagni P, Di Leo A, Saragoni L, et al; Italian Research Group for Gastric Cancer. Different patterns of recurrence in gastric cancer depending on Lauren's histological type: longitudinal study. World J Surg. 2002;26:1160-5.

21. Roviello F, Marrelli D, de Manzoni G, Morgagni P, Di Leo A, Saragoni L, et al; Italian Research Group for Gastric Cancer. Prospective study of peritoneal recurrence after curative surgery for gastric cancer. $\mathrm{Br} \mathrm{J}$ Surg. 2003;90:1113-9.

22. Kamei T, Kitayama J, Yamashita H, Nagawa H. Intraoperative blood loss is a critical risk factor for peritoneal recurrence after curative resection of advanced gastric cancer. World J Surg. 2009;33:1240-6.

23. Satoh Y, Mori K, Kitano K, Kitayama J, Yokota H, Sasaki $\mathrm{H}$, et al. Analysis for the combination expression of
CK20, FABP1 and MUC2 is sensitive for the prediction of peritoneal recurrence in gastric cancer. Jpn J Clin Oncol. 2012;42:148-52.

24. Okada K, Fujiwara Y, Nakamura Y, Takiguchi S, Nakajima K, Miyata H, et al. Oncofetal protein, IMP-3, a potential marker for prediction of postoperative peritoneal dissemination in gastric adenocarcinoma. J Surg Oncol. 2012;105:780-5.

25. Marutsuka T, Shimada S, Shiomori K, Hayashi N, Yagi $\mathrm{Y}$, Yamane T, et al. Mechanisms of peritoneal metastasis after operation for non-serosa-invasive gastric carcinoma: an ultrarapid detection system for intraperitoneal free cancer cells and a prophylactic strategy for peritoneal metastasis. Clin Cancer Res. 2003;9:678-85.

26. Peritoneal Carcinomatosis: Principles of management 1996. Pag 166. Paul Sugarbaker.

27. Cascinu S, Labianca R, Barone C, Santoro A, Carnaghi $\mathrm{C}$, Cassano A, et al. Adjuvant treatment of high-risk, radically resected gastric cancer patients with 5-fluorouracil, leucovorin, cisplatin and Epidoxorubibin in a randomized controlled trial. J Natl Cancer Inst doi: 10.1093/jnci/ djk131, 2007.

28. González-Moreno S. Peritoneal dissemination: a pending issue in gastric cancer worth exploring. Ann Surg Oncol. 2009;16:3217-8.

29. Jones RB, Myers CE, Guarino AM, Dedrick RL, Hubbard SM, DeVita VT. High volume intraperitoneal chemotherapy ("belly bath") for ovarian cancer. Pharmacologic basis and early results. Cancer Chemother Pharmacol. 1978;1:161-6.

30. Haley ES, Au GG, Carlton BR, Barry RD, Shafren DR. Regional administration of oncolytic Echovirus 1 as a novel therapy for the peritoneal dissemination of gastric cancer. J Mol Med (Berl). 2009;87:385-99.

31. Yano M, Yasuda T, Fujiwara Y, Takiguchi S, Miyata H, Monden M. Preoperative intraperitoneal chemotherapy for patients with serosa-infiltrating gastric cancer. J Surg Oncol. 2004;88:39-43.

32. Hagiwara A, Sawai K, Sakakura C, Shirasu M, Ohgaki M, Yamasaki J, et al. Complete omentectomy and extensive lymphadenectomy with gastrectomy improves the survival of gastric cancer patients with metastases in the adjacent peritoneum. Hepatogastroenterol. 1998;45:1922-9.

33. Fujita J, Kurokawa Y, Sugimoto T, Miyashiro I, Iijima S, Kimura Y, et al. Survival benefit of bursectomy in patients with resectable gastric cancer: interim analysis results of a randomized controlled trial. Gastric Cancer 2012;15:42-8

34. Weisberger AS, Levine B, Storaasli JP. Use of nitrogen mustard in treatment of serous effusions of neoplastic origin. JAMA 1955;159:1704-7.

35. Jaaback K, Johnson N. Intraperitoneal chemotherapy for the initial management of primary epithelial ovarian cancer. Cochrane Database Sys Rev CD005340, 2006.

36. Verwall VJ, van Ruth S, de Bree E. Randomised trial 
of cytoreduction and hyperthermic intraperitoneal chemotherapy versus systemic chemotherapy and palliative surgery in patients with peritoneal carcinomatosis of colorectal cancer. J Clin Oncol. 2003;21:3737-43.

37. Yan TD, Cao CQ, Munkholm-Larsen S. A pharmacological review on intraperitoneal chemotherapy for peritoneal malignancy. World J Gastr Oncol. 2010;2:10916.

38. Sugarbaker PH. Early postoperative intraperitoneal Adriamycin as an adjunctive treatment for advanced gastric cancer with lymph node or serosal invasion. In: Sugarbaker PH, ed Management of Gastric Cancer. Boston: Kluwer Academic Publishers, 1991:277-84.

39. Yan TD, Black D, Sugarbaker PH, Zhu J, Yonemura Y, Petrou G, et al. A Systematic Review and Meta-analysis of the Randomized Controlled Trials on Adjuvant Intraperitoneal Chemotherapy for Resectable Gastric Cancer. Ann Surg Oncol. 2007;14:2702-13.

40. Hamazoe R, Maeta M, Kaibara N. Intraperitoneal thermochemotherapy for prevention of peritoneal recurrence of gastric cancer-final results of a randomised controlled study. Cancer 1994;73:2048-52.

41. Fujimoto S, Takahashi M, Mutou T, Kobayashi K, Toyosawa T. Successful intraperitoneal hyperthermic chemoperfusion for the prevention of postoperative peritoneal recurrence in patients with advanced gastric carcinoma. Cancer 1999;85:529-34.

42. Mi DH, Li Z, Yang KH, Cao N, Lethaby A, Tian JH, et al. Surgery combined with intraoperative hyperthermic intraperitoneal chemotherapy (IHIC) for gastric cancer: A systematic review and meta-analysis of randomised controlled trial. Int J Hyperthermia 2013;29:156-67.

43. Kuramoto M, Shimada S, Ikeshima S, Matsuo A, Yagi Y, Matsuda M, et al. Extensive intraoperative peritoneal lavage as a standard prophylactic strategy for peritoneal recurrence in patients with gastric cancer. Ann Surg.
2009;250:242-6.

44. Yu XF, Ren ZG, Xue YW, Song HT, Wei YZ, Li CM. D2 lymphadenectomy can disseminate tumor cells into peritoneal cavity in patients with advanced gastric cáncer. Neoplasma 2013;60:174-81.

45. Yamamoto K, Shimada S, Hirota M, Yagi Y, Matsuda M, Baba H. EIPL (extensive intraoperative peritoneal lavage) therapy significantly reduces peritoneal recurrence after pancreatectomy in patients with pancreatic cáncer. Int J Oncol. 2005;27:1321-8.

46. Chang YM, Hsu KF, Yu JC, Chan DC, Chen CJ, Chen $\mathrm{TW}$, et al. Distilled water peritoneal lavage in patients with rupture hepatocellular carcinoma. Hepatogastroenterol. 2013;60:140-3.

47. Ito F, Camoriano M, Seshadri M, Evans SS, Kane JM 3rd, Skitzki JJ. Water: a simple solution for tumor spillage. Ann Surg Oncol. 2011;18:2357-63.

48. Viñuela EF, Gonen M, Brennan MF, Coit DG, Strong VE. Laparoscopic versus open distal gastrectomy for gastric cancer: a meta-analysis of randomized controlled trials and high-quality nonrandomized studies. Ann Surg. 2012;255:446-56.

49. Hao YX, Zhong H, Yu PW, Qian F, Zhao YL, Shi Y, et al. Influence of laparoscopic gastrectomy on the detection rate of gastric cancer cells in the peritoneal cavity. Ann Surg Oncol. 2010;17:65-72.

50. Sammartino P, Sibio S, Biacchi D, Cardi M, Accarpio F, Mingazzini P, et al. Prevention of Peritoneal Metastases from Colon Cancer in High-Risk Patients: Preliminary Results of Surgery plus Prophylactic HIPEC. Gastroenterology Res Pract. 2012;2012:141585. doi: 10.1155/2012/141585. Epub 2012 May 8.

51. Maehara Y, Hasuda S, Koga T, Tokunaga E, Kakeji Y, Sugimachi K. Postoperative outcome and sites of recurrence in patients of following curative resection of gastric cancer. Br J Surg. 2003;87:353-7. 